\title{
Radiofrequency Bias Correction of Magnetization Prepared Rapid Gradient Echo MRI at 7.0 Tesla Using an External Reference in a Sequential Protocol
}

\author{
Hampus Olsson ${ }^{1, *} \mathbb{D}$, Mikael Novén ${ }^{2} \mathbb{D}$, Jimmy Lätt ${ }^{3}$, Ronnie Wirestam ${ }^{1} \mathbb{D}$ and Gunther Helms ${ }^{1} \mathbb{D}$ \\ 1 Department of Medical Radiation Physics, Clinical Sciences Lund, Lund University, 22185 Lund, Sweden; \\ ronnie.wirestam@med.lu.se (R.W.); gunther.helms@med.lu.se (G.H.) \\ 2 Department of Linguistics and Phonetics, Lund University, 22100 Lund, Sweden; mikael.noven@gmail.com \\ 3 Center for Medical Imaging and Physiology, Skåne University Hospital, 22100 Lund, Sweden; \\ jimmy.latt@med.lu.se \\ * Correspondence: hampus.olsson@med.lu.se
}

check for updates

Citation: Olsson, H.; Novén, M.; Lätt, J.; Wirestam, R.; Helms, G. Radiofrequency Bias Correction of Magnetization Prepared Rapid Gradient Echo MRI at 7.0 Tesla Using an External Reference in a Sequential Protocol. Tomography 2021, 7, 434-451. https://doi.org/10.3390/

tomography7030038

Academic Editor: Bradford A. Moffat

Received: 15 July 2021

Accepted: 9 September 2021

Published: 13 September 2021

Publisher's Note: MDPI stays neutral with regard to jurisdictional claims in published maps and institutional affiliations.

Copyright: (c) 2021 by the authors. Licensee MDPI, Basel, Switzerland. This article is an open access article distributed under the terms and conditions of the Creative Commons Attribution (CC BY) license (https:// creativecommons.org/licenses/by/ $4.0 /)$.

\begin{abstract}
At field strengths of $7 \mathrm{~T}$ and above, $T_{1}$-weighted imaging of human brain suffers increasingly from radiofrequency (RF) $B_{1}$ inhomogeneities. The well-known MP2RAGE (magnetization prepared two rapid acquisition gradient echoes) sequence provides a solution but may not be readily available for all MR systems. Here, we describe the implementation and evaluation of a sequential protocol to obtain normalized magnetization prepared rapid gradient echo (MPRAGE) images at 0.7, 0.8, or 0.9-mm isotropic spatial resolution. Optimization focused on the reference gradient-recalled echo (GRE) that was used for normalization of the MPRAGE. A good compromise between whitegray matter contrast and the signal-to-noise ratio (SNR) was reached at a flip angle of $3^{\circ}$ and total scan time was reduced by increasing the reference voxel size by a factor of 8 relative to the MPRAGE resolution. The average intra-subject coefficient-of-variation $(\mathrm{CV})$ in segmented white matter (WM) was $7.9 \pm 3.3 \%$ after normalization, compared to $20 \pm 8.4 \%$ before. The corresponding inter-subject average $C V$ in WM was $7.6 \pm 7.6 \%$ and $13 \pm 7.8 \%$. Maps of $T_{1}$ derived from forward signal modelling showed no obvious bias after correction by a separately acquired flip angle map. To conclude, a non-interleaved acquisition for normalization of MPRAGE offers a simple alternative to MP2RAGE to obtain semi-quantitative purely $T_{1}$-weighted images. These images can be converted to $T_{1}$ maps, analogously to the established MP2RAGE approach. Scan time can be reduced by increasing the reference voxel size which has only a miniscule effect on image quality.
\end{abstract}

Keywords: bias correction; intensity correction; MPRAGE; MP2RAGE; B1; 7T; ultra-high field; longitudinal relaxation; T1; T1-mapping

\section{Introduction}

The magnetization prepared rapid gradient echo (MPRAGE) sequence has become the standard for structural $T_{1}$-weighted $\left(T_{1}-\mathrm{w}\right) 3 \mathrm{D}$ imaging. The $T_{1}$ contrast is obtained by an inversion pulse followed by a rapid gradient echo (RAGE) readout with optional delays for free recovery before and after the readout [1]. At ultra-high field (UHF) MRI (7 T or above), the signal-to-noise ratio (SNR) is improved through the increased polarization of nuclear spins, which can be translated into either increased spatial resolution or faster scan times. The former alternative allows for visualization of substructures, unfeasible at lower field strengths [2]. A major challenge of UHF is the increased inhomogeneity of the radiofrequency $(\mathrm{RF}) B_{1}$ field, which varies based on subject, positioning, and coil [3]. Because of the unpredictability of the $B_{1}$ field, pixel values from images acquired at different scanning sessions are generally not reproducible. This means that an important requirement for longitudinal or multi-site studies is not fulfilled. The increased inhomogeneity applies to both the transmit $\left(B_{1}^{+}\right)$and the receive sensitivity components, which combine to form a 
total bias field, that diminishes the uniformity of the image. To correct for this intensity bias, it was suggested to acquire a reference gradient-recalled echo (GRE) in conjunction with the MPRAGE sequence [4]. Through a simple division of the two acquisitions, a normalized MPRAGE image is obtained where signal variations due to the shared receive coils are eliminated, thus improving image quality as well as reproducibility. $B_{1}{ }^{+}$affects the MPRAGE and the GRE pulse sequences differently through the local flip angle and, therefore, the related bias is reduced but not removed in the normalized MPRAGE. The division also removes the influence of proton density (PD) and $T_{2}{ }^{*}$, thereby creating a purely $T_{1}-\mathrm{w}$ image with improved tissue contrast. As the pixel values depend on the sequence, the technique is considered to be "semi-quantitative". The reference GRE can be acquired either separately or interleaved with the MPRAGE at a longer inversion time (TI), and the latter approach has been popularized as the MP2RAGE (magnetization prepared two rapid acquisition gradient echoes) sequence [5].

In this work, we describe an implementation where MPRAGE and reference are acquired in a non-interleaved, sequential fashion to produce the normalized MPRAGE. This approach is an accessible alternative to the interleaved MP2RAGE which, initially, was not readily available on our 7T MR system. Although the non-interleaved variant is expected to be more susceptible to inter-scan subject motion, the reference GRE can be accelerated through enlarged acquisition voxels, allowing for shorter scan time. The optimization procedure focused on the reference GRE, specifically on the flip angle and voxel size, in an effort to improve contrast, $\mathrm{SNR}$, residual $B_{1}{ }^{+}$bias, and scan time. The protocol was implemented at three different spatial resolutions, and normalized MPRAGE images acquired at a higher versus a lower spatial resolution were compared. The intra-subject and intersubject reproducibility (crucial for a semi-quantitative protocol) were also investigated. Finally, the feasibility of calculating $T_{1}$ maps from a look-up table (LUT) of the normalized signal, obtained by forward modelling, was explored. The latter approach extends beyond the semi-quantitative domain and results in fully quantitative maps which would allow for a more direct biophysical interpretation in terms of, for instance, myelination [6]. It is analogous to the approach described for the interleaved MP2RAGE by Marques et al. [5]. The work presented here documents an optimized protocol for bias field-corrected structural imaging at $7 \mathrm{~T}$, easily implemented and interpretable by radiologists.

\section{Materials and Methods}

\subsection{Theory}

The influence of receive sensitivity, $\mathrm{PD}$, and $T_{2}{ }^{*}$ were removed through division of the MPRAGE signal, $S_{M P}$, by the reference GRE signal, $S_{\mathrm{GRE}}$, to yield the normalized MPRAGE

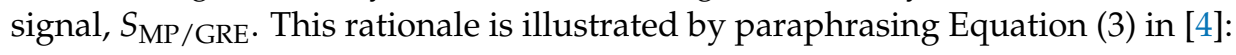

$$
S_{\mathrm{MP} / \mathrm{GRE}}=\frac{S_{\mathrm{MP}}}{S_{\mathrm{GRE}}} \propto \frac{f_{\mathrm{R}} \rho \widetilde{M}_{\mathrm{z}, \mathrm{MP}} \sin \left(f_{\mathrm{T}} \alpha_{\mathrm{MP}}\right) \exp \left(-T_{\mathrm{E}} R_{2}^{*}\right)}{f_{\mathrm{R}} \rho \widetilde{M}_{\mathrm{z}, \mathrm{GRE}} \sin \left(f_{\mathrm{T}} \alpha_{\mathrm{GRE}}\right) \exp \left(-T_{\mathrm{E}} R_{2}^{*}\right)}=\frac{\widetilde{M}_{\mathrm{z}, \mathrm{MP}} \sin \left(f_{\mathrm{T}} \alpha_{\mathrm{MP}}\right)}{\widetilde{M}_{\mathrm{z}, \mathrm{GRE}} \sin \left(f_{\mathrm{T}} \alpha_{\mathrm{GRE}}\right)}
$$

where $\rho$ denotes $\mathrm{PD}, \widetilde{M}_{z, \mathrm{MP}, \mathrm{GRE}}=M_{\mathrm{z}, \mathrm{MP}, \mathrm{GRE}} / \rho$ is the longitudinal magnetization per unit $\mathrm{PD}, f_{\mathrm{R}}$ is a factor accounting for the spatial dependence of receive sensitivity (here, a weighted linear combination of individual channels). The arguments of the sine functions are the local flip angles, i.e., $\alpha_{\mathrm{loc}, \mathrm{MP}, \mathrm{GE}}=f_{\mathrm{T}} \alpha_{\mathrm{MP}, \mathrm{GE}}$, where $f_{\mathrm{T}}$ takes account of the transmit field $\left(B_{1}^{+}\right)$inhomogeneity. Finally, $R_{2}^{*}=1 / T_{2}^{*}$ is the effective transverse relaxation rate. Note that $\widetilde{M}_{\mathrm{Z}, \mathrm{MP}}$ is acquired under transient conditions towards a driven equilibrium, $\widetilde{M}_{0}^{*}$, with an increased rate $R_{1}^{*}=1 / T_{1}^{*}[7]$ :

$$
\widetilde{M}_{0}^{*}=\widetilde{M}_{0} \cdot \frac{1-\exp \left(-R_{1} T R\right)}{1-\exp \left(-R_{1}^{*} T R\right)}
$$

where $\widetilde{M}_{0}$ is the magnetization at thermal equilibrium, $R_{1}^{*}$ is

$$
R_{1}^{*}=R_{1}-\ln \left(\cos \left(f_{T} \alpha_{\mathrm{MP}}\right)\right) / T R,
$$


and $R_{1}=1 / T_{1}$. If full relaxation within one cycle is not obtained, $\widetilde{M}_{z, \mathrm{MP}}$ will attain a dynamic steady-state between cycles, usually occurring after a few cycles [8]. The degree of agreement between $\widetilde{M}_{\mathrm{z}, \mathrm{MP}}$ and $\widetilde{M}_{0}^{*}$ when the central $\mathrm{k}$-space line is acquired is a function of TI and $T_{1}$ as well as of the local flip angle and thus $f_{\mathrm{T}}$. Note that, without an inversion pulse, $\widetilde{M}_{z, G R E}$ is always acquired under steady-state conditions so that

$$
\widetilde{M}_{\mathrm{z}, \mathrm{GRE}}=\widetilde{M}_{0}^{*}\left(f_{\mathrm{T}} \alpha_{\mathrm{GRE}}, T_{1}\right) .
$$

Thus, transmit field related bias may not be removed completely for $\alpha_{\mathrm{MP}}=\alpha_{\mathrm{GRE}}$ as Equation (1) might imply.

\subsection{Equipment}

The protocols were implemented on an actively shielded 7T MR system (Achieva, Philips Healthcare, Best, The Netherlands), using a head coil with two transmitter channels and 32 receive channels (Nova Medical, Wilmington, MA, USA). Healthy subjects were scanned after giving informed written consent and the study was approved by the regional Ethical Review Board. Dielectric pads were used in the experiments [9].

\subsection{MPRAGE Acquisition}

The MPRAGE protocol was built upon the standard protocol for structural MRI available at the research site. Isotropic voxel sizes of either $0.7^{3}, 0.8^{3}$, or $0.9^{3} \mathrm{~mm}^{3}$ were acquired with a slab-selective excitation, a readout flip angle of $\alpha_{\mathrm{MP}}=8^{\circ}$, TR $=8 \mathrm{~ms}$, fatwater in-phase TE $=1.97 \mathrm{~ms}$ and a bandwidth/px of $503 \mathrm{~Hz} / \mathrm{px}$. For inversion, an adiabatic pulse with duration $22 \mathrm{~ms}$ and a maximal $B_{1}$ amplitude of $15 \mu \mathrm{T}$ was used. The delay from inversion to the central k-space readout (linear phase encoding) was TI $=1200 \mathrm{~ms}$ and the time between inversions was $\mathrm{T}_{\text {cycle }}=3500 \mathrm{~ms}$. These timings (i) allowed for a period of free relaxation after the readout train (increasing dynamic range) and (ii) ensured that the $M_{\mathrm{z}}$ of cerebrospinal fluid (CSF) was close to the zero-crossing during acquisition of the center of k-space. Both (i) and (ii) will improve $T_{1}$ contrast in magnitude images. After each inversion, a single $2 \mathrm{D}$ plane of $\mathrm{k}$-space was acquired so that the turbo factor (TF) was identical to the acquisition matrix size in the inner loop phase-encoded direction, $N_{y}$. Parallel imaging in the form of sensitivity encoding (SENSE) was applied in the outer loop (right-left direction) with a reduction factor of 2.5 [10]. The inner loop corresponded to the anterior-posterior (AP) direction. Switching between the different resolutions will alter TF and thus affect the $T_{1}$ contrast (see Results 4.3$)$. For $(0.7 \mathrm{~mm})^{3}$ resolution, a small inner loop SENSE reduction factor of 1.11 had to be applied to fit the readout train within TI $=1200 \mathrm{~ms}$ $\left(\mathrm{TF}=N_{\mathrm{y}} / 1.11\right)$. Note that the FOV in the outer loop $\left(\mathrm{FOV} \mathrm{FH}_{\mathrm{AP}, \mathrm{RL}}=230 \times 230 \times 180 \mathrm{~mm}^{3}\right)$ can be enlarged without affecting contrast. SENSE-related wrap-around artifacts in the AP direction were avoided by an oversampling margin (default setting) which did not affect the acquisition time $\left(T_{\text {acq }}\right)$. Lastly, to explore the possibility of further reducing $T_{\text {acq, }}$ the protocol was implemented with elliptical k-space sampling in the phase encoding directions. A prerequisite for this kind of readout is a multi-shot acquisition combined with a zigzag k-space trajectory involving both phase encoding directions during the RAGE readout. This switching between the inner and outer phase encoding loops is performed in such a way that overall contrast is unaffected for constant TF. The acquisition matrix $\left(N_{\mathrm{x}, \mathrm{y}, \mathrm{z}}\right)$, $\mathrm{TF}$ and $T_{\text {acq }}$ for all spatial resolutions with/without elliptical $\mathrm{k}$-space phase encoding are listed in Table 1. 
Table 1. Acquisition times $\left(T_{\text {acq }}\right)$ of the non-interleaved protocol at different spatial resolutions and with/without elliptical k-space phase encoding. The MPRAGE with $(0.7 \mathrm{~mm})^{3}$ resolution used an inner loop SENSE $_{\mathrm{AP}}=1.11$ to accommodate the readout train.

\begin{tabular}{|c|c|c|c|}
\hline Parameter/Resolution & $(0.7 \mathrm{~mm})^{3}$ & $(0.8 \mathrm{~mm})^{3}$ & $(0.9 \mathrm{~mm})^{3}$ \\
\hline $\operatorname{MP} N_{x, y, z}$ & $328 \times 328 \times 257$ & $288 \times 288 \times 225$ & $256 \times 256 \times 200$ \\
\hline GRE $N_{x, y, z}$ & $164 \times 164 \times 128$ & $144 \times 144 \times 112$ & $128 \times 128 \times 100$ \\
\hline MP TF & 296 & 288 & 256 \\
\hline $\mathrm{MP} T_{\mathrm{acq}}(\mathrm{min})$ & $05: 59$ & $05: 14$ & 04:39 \\
\hline$M P T_{\text {acq }}$ (elliptical) (min) & $04: 35$ & 04:04 & $03: 36$ \\
\hline GRE $T_{\mathrm{acq}}(\mathrm{min})$ & 01:10 & $00: 53$ & $00: 42$ \\
\hline GRE $T_{\text {acq }}$ (elliptical) (min) & $00: 54$ & $00: 40$ & $00: 32$ \\
\hline Total $T_{\text {acq }}(\mathrm{min})$ & 07:09 & 06:07 & $05: 21$ \\
\hline Total $T_{\text {acq }}$ (elliptical) (min) & $05: 45$ & $04: 57$ & 04:18 \\
\hline
\end{tabular}

\subsection{Reference GRE Acquisition}

The steady-state GRE sequence was acquired with TR, TE, and outer loop SENSE factor identical to the MPRAGE sequence, but with $50 \%$ zero-filling in the outer loop to reduce scan time. In other words, voxel dimensions in the right-left direction were 1.4, 1.6, or $1.8 \mathrm{~mm}$ for the $(0.7)^{3},(0.8)^{3}$, and $(0.9)^{3} \mathrm{~mm}^{3}$ protocols, respectively. When determining the in-plane voxel size (see Results 3.2), the bandwidth/pixel was changed accordingly so that the absolute fat signal displacement was constant between GRE and MPRAGE. Receiver gain and flip angle were calibrated for MPRAGE and then kept constant during the GRE. After acquisition, the volume was reconstructed to the same matrix size as the MPRAGE through zero-filling.

\subsection{Data Post-processing}

Images in the Digital Imaging and Communications in Medicine (DICOM) file format were exported, pseudo-anonymized, and converted to the Neuroimaging Informatics Technology Initiative (NIfTI) file format using an in-house modification of the dcm2niix tool [11]. The platform-dependent scaling of signal intensities was reverted from stored values to 32-bit floating point/1000 (to obtain pixel values in the range of 0-1000) [12]. Spatial dimensions were re-ordered to transverse orientation according to radiological convention (right-left). Rigid co-registration of the reference GRE to the MPRAGE volume was performed using the FMRIB Linear Image Registration Tool (FLIRT) [13,14], where after the normalization was performed as in Equation (1). A mask of the brain was obtained by applying the Brain Extraction Tool (BET) to the PD-w reference GRE [15]. Segmentation of the three major tissue classes, white matter (WM), gray matter (GM) and CSF, was performed using the FMRIB Automated Segmentation Tool (FAST) [16]. Improvement in spatial homogeneity, after signal normalization, for different parameter settings of the reference GRE was analyzed using the coefficient-of-variation $(\mathrm{CV})$ over the tissue classes in the normalized MPRAGE. The $C V$ within a small WM ROI was used to evaluate relative changes in SNR; the rationale for this approach was that the $C V$ in a spatially restricted ROI of homogenous tissue should be unaffected by $\mathrm{B}_{1}{ }^{+}$and dominated by SNR. Average contrast between WM and GM was defined as $C=\frac{\bar{S}_{\mathrm{WM}}-\bar{S}_{\mathrm{GM}}}{\bar{S}_{\mathrm{WM}}+\bar{S}_{\mathrm{GM}}}$ where $\bar{S}_{\mathrm{WM}}$ and $\bar{S}_{\mathrm{GM}}$ is the average pixel value of the respective segmented tissue type.

\subsection{Readout Flip Angle of Reference GRE}

A higher $\alpha_{\text {GRE }}$ will increase the $T_{1}-w$ of the predominantly PD-w reference GRE, thus reducing tissue contrast in the normalized MPRAGE image. On the other hand, reducing $\alpha_{\text {GRE }}$ below the Ernst angle will decrease SNR. To find a compromise between SNR and tissue contrast in the normalized MPRAGE, $\alpha_{\text {GRE }}$ was varied from $1^{\circ}$ to $6^{\circ}$ in increments 
of $1^{\circ}$ in a single subject. In order to obtain comparable pixel values, reference GREs were scaled by a global factor giving a scaled signal

$$
S_{\mathrm{GRE}, \mathrm{scaled}}\left(\alpha_{\mathrm{GRE}, \mathrm{i}}\right)=S_{\mathrm{GRE}}\left(\alpha_{\mathrm{GRE}, \mathrm{i}}\right) /\left(\alpha_{\mathrm{GRE}, \mathrm{i}} \frac{0.5 \cdot \alpha_{\mathrm{GRE}, 6}^{2}+R_{1} T R}{\alpha_{\mathrm{GRE}, 6}\left(0.5 \alpha_{\mathrm{GRE}, \mathrm{i}}^{2}+R_{1} T R\right)}\right),
$$

where a single $R_{1}=0.83 \mathrm{~s}^{-1}$ (corresponding to expected WM $T_{1} \approx 1.2 \mathrm{~s}$ ) was used to approximate the saturation of $M_{\mathrm{z}}[17,18]$. The scaling only served to facilitate identical windowing of the images and does not affect the analysis itself. The contrast between segmented WM and GM as well as the $C V$ of segmented WM was plotted as a function of $\alpha_{\mathrm{GRE}}$. The latter was used as a proxy to evaluate any residual influence of $B_{1}{ }^{+}$inhomogeneities.

\subsection{Voxel Size of Reference GRE}

The $B_{1}$-related intensity bias (of both receive and transmit effects) comprises mostly low spatial frequencies. Thus, a reference GRE with low spatial resolution is sufficient to correct for $B_{1}$ inhomogeneity. On the other hand, this is not the case for PD and $T_{2}{ }^{*}$ contrast. If the normalized MPRAGE is purposed to produce only semi-quantitative images with a greatly reduced intensity field bias, some dilution of the "pure" $T_{1}$ contrast could be acceptable to reduce scan time. In an effort to further reduce scan time and evaluate the effect on the resulting image quality, an MPRAGE volume with $0.7 \mathrm{~mm}$ isotropic resolution was normalized by a reference GRE for which the voxel size, $V_{\text {ref, }}$ was varied in-plane as $0.70 \times 0.70,1.05 \times 1.05,1.40 \times 1.40,2.10 \times 2.10$, and $2.80 \times 2.80 \mathrm{~mm}^{2}$ (i.e., $\times 1, \times 1.5, \times 2$, $\times 3, \times 4$ the MPRAGE resolution) in a single subject. The voxel dimension in the outer loop (right-left direction) was constant at $1.40 \mathrm{~mm}$ resulting in acquisition times of $T_{\mathrm{acq}}=2: 21$, 1:35, 1:10, 0:49, and 0:37 $\mathrm{min}$, respectively.

\subsection{Implementation at Different Resolutions}

Two finalized protocols (Results 3.1-3.2) with voxel sizes of $(0.7 \mathrm{~mm})^{3}$ and $(0.9 \mathrm{~mm})^{3}$ were used on a single subject to compare the effect of the corresponding TF on the contrast. Further, the potential increase of interpolation artifacts at different spatial resolutions (especially in the reference GRE) was of interest to assess.

\subsection{Intra-Subject Variability}

One subject (male, 51 years old, body mass index (BMI) of 23.6) was scanned on five separate occasions over a period of about 8 months using the normalized MPRAGE protocol with $0.7 \mathrm{~mm}$ isotropic resolution. Maps of $C V$ were compared before and after normalization and the average $C V s$ in $\mathrm{WM}, \mathrm{GM}$, and CSF were calculated. Regions of interest (ROIs) were manually delineated in the $C V$ maps to study local variability. The ROIs were defined in left frontal WM, the left caudate head, the cerebellum, and the left temporal lobe.

\subsection{Inter-Subject Variability}

The $0.8 \mathrm{~mm}$ isotropic resolution protocol was previously used in a separate work to study correlations between cortical morphology and language-learning aptitude [19]. From the underlying subject population of this work, a subset of 10 randomly chosen volunteers ( 7 female, $22 \pm 1.9$ years old) were used to examine the inter-subject variability of the normalized MPRAGE images. The subjects were all of normal build, that is, height, weight, and BMI were perceived to fall within the 5th and 95th percentiles. Images were diffeomorphically registered to the Montreal Neurological Institute (MNI) space using the diffeomorphic anatomical registration using exponentiated Lie algebra (DARTEL) algorithm as embedded in the statistical parameter mapping (SPM)-based histological MRI (hMRI) toolbox [20,21]. As in the previous subsection, maps of the $C V$ were then compared before and after normalization and the average CVs in WM, GM, and CSF were calculated. ROIs were likewise defined in MNI space in the same four areas as 
in the previous subsection, i.e., left frontal WM, left caudate head, cerebellum, and the left temporal lobe. The average CVs in WM, GM and CSF were also calculated based on the average pixel intensities of individual segmentations rather than one common segmentation in MNI space.

\subsection{Comparison to MP2RAGE}

The finalized $(0.7 \mathrm{~mm})^{3}$ sequential protocol was compared to an interleaved MP2RAGE protocol with the same spatial resolution in one subject [22]. The pulse sequence parameters of the MP2RAGE protocol was as follows: $T_{\text {cycle }} / T_{1} / T_{2}=5 / 0.9 / 2.75 \mathrm{~s}, \alpha_{1} / \alpha_{2}=5 / 3$ degrees, $\mathrm{TF}=256, \mathrm{TR} / \mathrm{TE}=6.8 / 2.4 \mathrm{~ms}$, bandwidth $/ \mathrm{px}=365 \mathrm{~Hz}, N_{\mathrm{x}, \mathrm{y}, \mathrm{z}}=320 \times 320 \times 256$, $T_{\text {acq }}=8: 20$ min with a partial Fourier acquisition of 0.75, and a SENSE-factor of 2 in the outer loop right-left direction as well as elliptical k-space phase encoding. The same inversion pulse as in the sequential protocol was used. The MP2RAGE image was calculated using complex data as described in ref. [5] and rigidly co-registered to the sequentially normalized MPRAGE image.

\subsection{2. $T_{1}$ Calculation}

For proof-of-principle, $T_{1}$-mapping using a LUT-based approach was performed on a healthy subject using the $(0.8 \mathrm{~mm})^{3}$ resolution protocol together with a DREAM flip angle map [23]. First, the evolution of the longitudinal magnetization, $M_{z}$, was simulated for the MPRAGE sequence with imaging parameters as described above, i.e., with $\alpha_{\mathrm{MP}}=8^{\circ}$, $\mathrm{TR}=8 \mathrm{~ms}, \mathrm{TI}=1200 \mathrm{~ms}$ and $\mathrm{TF}=288$.

The evolution of $M_{z}$ during $T_{\text {cycle }}$ in the outer loop steady-state (occurring after 2-3 cycles) was simulated using Equations (2) and (3) in the intervals where readout occurred and using normal $T_{1}$ relaxation where readout did not occur. The simulations can be performed with $\widetilde{M}_{z}$ (i.e., per unit $\rho$ ). The inversion efficiency applied the end of each cycle was assumed to be $f_{\text {inv }}=0.96$ [5]. The LUT-derived signal was then calculated for a constant $\alpha_{\mathrm{MP}}$ but over a range of $f_{\mathrm{T}}$ as:

$$
S_{\mathrm{MP}, \mathrm{LUT}}=\frac{\widetilde{M}_{z, \mathrm{MP}}\left(f_{\mathrm{T}}, T_{1}, T I\right)}{\widetilde{M}_{0}} \sin \left(f_{\mathrm{T}} \alpha_{\mathrm{MP}}\right) .
$$

The reference GRE was assumed to always be in the inner loop steady-state. Hence, $\widetilde{M}_{z, \mathrm{GRE}}$ and consequently the LUT-derived GRE signal, $S_{\mathrm{GRE}, \mathrm{LUT}}$, is constant for a constant $T_{1}$ and $f_{\mathrm{T}}$ :

$$
S_{\mathrm{GRE}, \mathrm{LUT}}=\frac{\widetilde{M}_{z, \mathrm{GRE}}\left(f_{\mathrm{T}}, T_{1}\right)}{\widetilde{M}_{0}} \sin \left(f_{\mathrm{T}} \alpha_{\mathrm{MP}}\right) .
$$

Thus, 2D $\left(n_{T_{1}} \times n_{f_{\mathrm{T}}}\right)$ LUTs of $S_{\mathrm{MP}, \mathrm{LUT}}$ and $S_{\mathrm{GRE}, \mathrm{LUT}}$ are obtained for a range of $1 \mathrm{~s} \leq T_{1} \leq 5 \mathrm{~s}$ (step size of $1 \mathrm{~ms}, n_{T_{1}}=5000$ ) and $0.4 \leq f_{\mathrm{T}} \leq 1.6$ (step size of 0.01, $\left.n_{f_{\mathrm{T}}}=121\right)$. The LUTs of the two simulated signals were then combined as follows [5]:

$$
S_{\mathrm{MP} 2 \mathrm{RAGE}, \mathrm{LUT}}\left(T_{1}, f_{\mathrm{T}}\right)=\frac{S_{\mathrm{MP}, \mathrm{LUT}} \cdot S_{\mathrm{GRE}, \mathrm{LUT}}}{S_{\mathrm{MP}, \mathrm{LUT}}^{2}+S_{\mathrm{GRE}, \mathrm{LUT}}^{2}} .
$$

Thus, the values are limited to $0 \leq S_{\text {MP2RAGE,LUT }}\left(T_{1}, f_{\mathrm{T}}\right) \leq 0.5$ in the final LUT for comparison with $S_{\mathrm{MP} 2 \mathrm{RAGE}}=\frac{S_{\mathrm{MP}} \cdot S_{\mathrm{GRE}}}{S_{\mathrm{MP}}^{2}+S_{\mathrm{GRE}}^{2}}$ calculated from the measured magnitude signals. Note that the measured $S_{\text {MP2RAGE }}\left(T_{1}, f_{T}\right)$ is limited to positive values for this implementation, since it is not possible to relate the phases of two signals measured sequentially. The $T_{1}$ for which $\left|S_{\text {MP2RAGE }}-S_{\text {MP2RAGE,LUT }}\right|$ was minimal was calculated pixelwise either for $f_{\mathrm{T}}=1$ or as determined by the DREAM flip angle map. 


\section{Results}

\subsection{Readout Flip Angle of Reference GRE}

There was a continuous decrease in tissue contrast in the normalized volume as $\alpha_{\text {GRE }}$ increased (Figure 1). This decrease was evident both from visual inspection (for $\alpha_{\mathrm{GRE}}>3^{\circ}$ ) and from the quantitative comparison of segmented WM and GM (panel C). The contrast was still increased after normalization, compared to before, for all values of $\alpha_{\text {GRE }}$. The ROI analysis (panel D) showed no decrease in $C V$, neither at lower $\alpha_{G R E}$ nor after normalization, and no apparent change in SNR could thus be identified. However, a minimum in the $C V$ of segmented $W M$ (panel E) was observed at $\alpha_{\mathrm{GRE}}=3^{\circ}$, implying minimal influence of residual $B_{1}{ }^{+}$inhomogeneities at this setting. The $B_{1}{ }^{+}$influence was visually identifiable as elevated pixel values in the center of the brain using $\alpha_{\mathrm{GRE}}=6^{\circ}$ (panel B). Based on these results, and to avoid deviating too far from the Ernst angle $\left(\sim 7^{\circ}-5^{\circ}\right.$ for $\left.1000 \mathrm{~ms} \leq T_{1} \leq 2000 \mathrm{~ms}\right), \alpha_{\mathrm{GRE}}=3^{\circ}$ was deemed optimal and chosen for the final protocol. At $\alpha_{\mathrm{GRE}}=3^{\circ}$, the WM-GM contrast was $C=0.18$ and the variability across segmented WM was $C V=9 \%$ after normalization compared to $C=0.10$ and $C V=34 \%$ before.

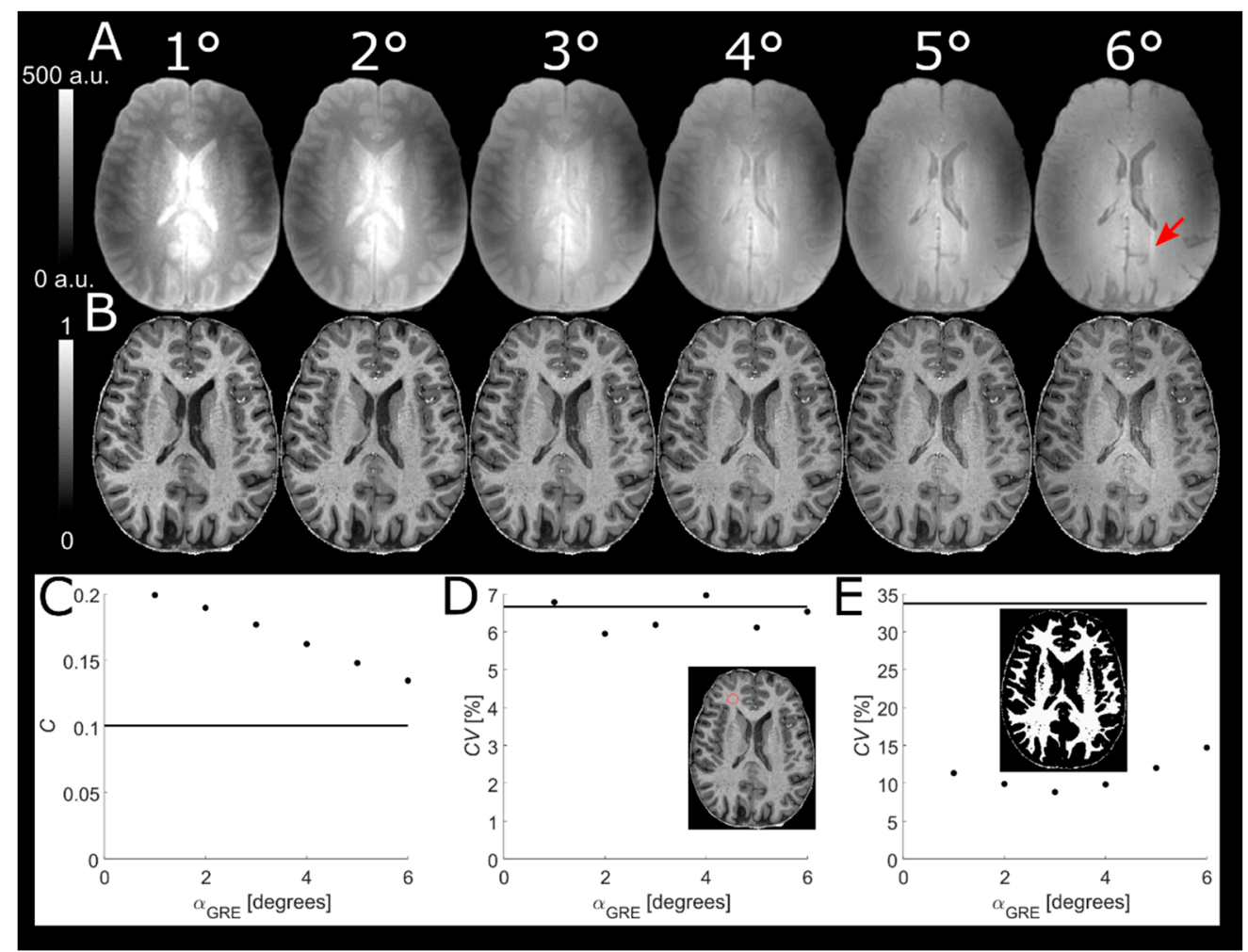

Figure 1. Reference GREs with different $\alpha_{\mathrm{GRE}}$ (top row, (A)) used to obtain normalized MPRAGE volumes (middle row, (B)). Decreasing WM-GM contrast with increasing $\alpha_{\mathrm{GRE}}$ is evident from visual inspection of the normalized volumes and verified in the scatter plot (C). No change in SNR could be identified by the $C V$ in a WM ROI (red circle) (D). The $C V$ in whole segmented WM had a minimum at $\alpha_{\mathrm{GRE}}=3^{\circ}$, implying minimum influence from residual $B_{1}{ }^{+}$effects $(\mathbf{E})$. These residual $B_{1}{ }^{+}$effects are visually identifiable as hyperintense pixels in the center of the normalized MPRAGE for $\alpha_{\mathrm{GRE}}=6^{\circ}(\mathrm{B})$. The solid lines in the scatter plots represent the respective MPRAGE volume values before normalization. In this experiment, the dielectric pads caused a visible fold-over artefact (red arrow).

\subsection{Voxel Size of Reference GRE}

Increasing $V_{\text {ref }}$ (Figure 2) yielded very similar normalized MPRAGE images (panel B). Although some ringing artifacts were visible in the images (cf. red arrow in panel B), increased $V_{\text {ref }}$ did not cause any noticeably stronger effect. No change in WM-GM contrast $\left(C=0.24\right.$ at $V_{\text {ref }}=0.7 \times 0.7 \times 1.4$ vs. $C=0.23$ at $\left.V_{\text {ref }}=2.8 \times 2.8 \times 1.4\right)$ was observed 
with increasing $V_{\text {ref }}$ (panel C). Although a higher SNR is to be expected for larger $V_{\text {ref }}$, no trend in the $C V$ of the WM ROI could be discerned (panel D). A weak trend of increasing $C V$ in segmented $\mathrm{WM}$ was observed $\left(C V=7.3 \%\right.$ at $V_{\text {ref }}=0.7 \times 0.7 \times 1.4$ vs. $C V=9.5 \%$ at $V_{\text {ref }}=2.8 \times 2.8 \times 1.4$ ), possibly reflecting partial volume effects (PVEs) (panel E). No difference between $V_{\text {ref }}=0.7 \times 0.7 \times 1.4$ and $V_{\text {ref }}=1.4 \times 1.4 \times 1.4$ was discernible. Hence, $V_{\text {ref }}$ in one dimension was set to twice that of the MPRAGE voxel size in one dimension, i.e., $1.4^{3} / 1.6^{3} / 1.8^{3} \mathrm{~mm}^{3}$ for $0.7^{3} / 0.8^{3} / 0.9^{3} \mathrm{~mm}^{3}$, which reduced the total scan time by $71 / 53 / 42$ s respectively (i.e., a reduction by $14 / 14 / 12 \%$ ). A higher $V_{\text {ref }}$ was not employed to avoid more pronounced PVEs and interpolation errors.

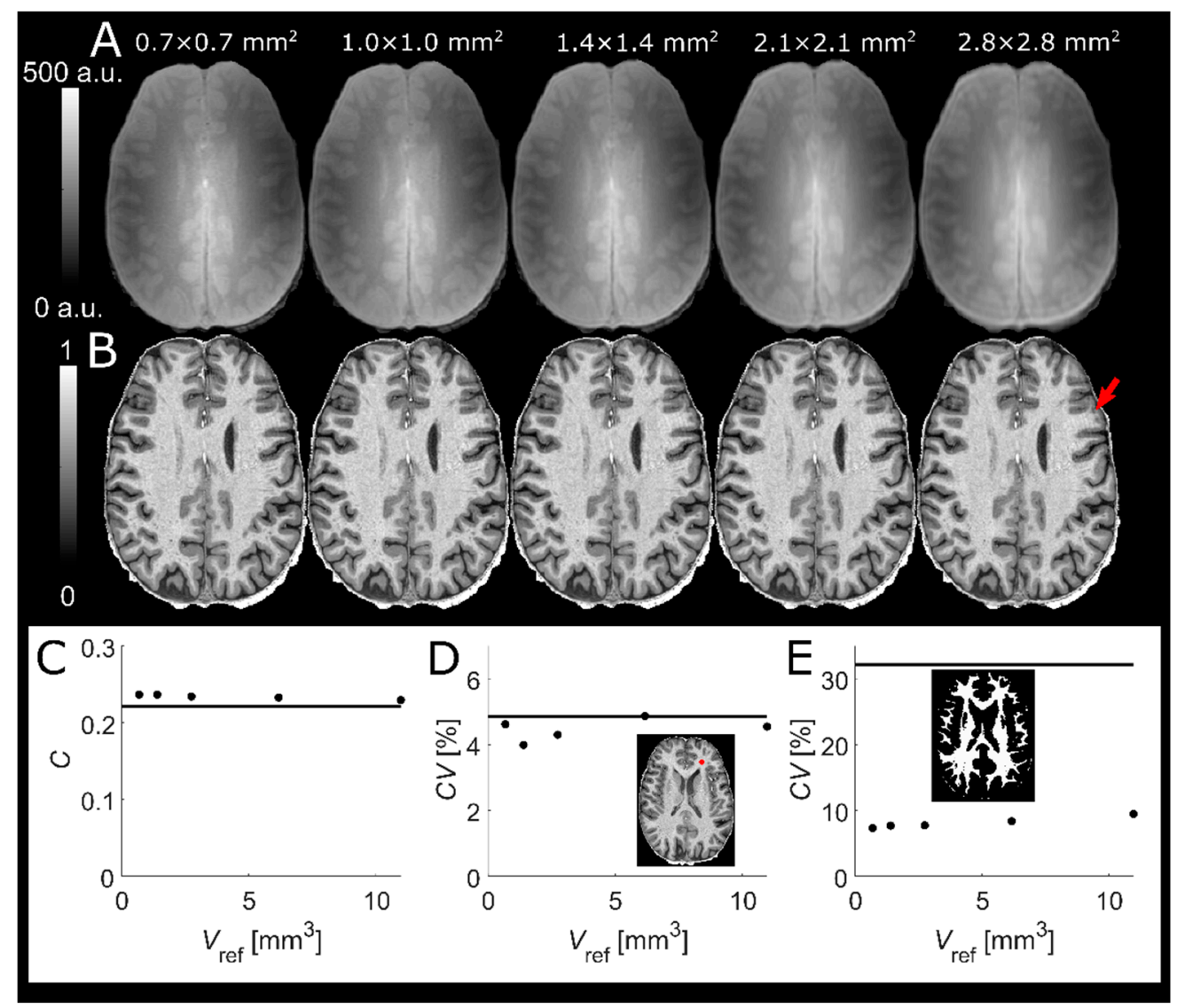

Figure 2. Reference GREs with different in-plane voxel sizes (top row, (A))) used to obtain normalized MPRAGE (middle row, (B)). The reference GREs and thereby also the normalized MPRAGE volumes are very similar. Some ringing artifacts are visible (red arrow) but do not appear to severely affect image quality even at the lowest resolution in this experiment. Scatter plot of contrast vs. voxel volume (C) shows no change in WM-GM contrast with increasing $V_{\text {ref }}$. No change in SNR could be identified by the $C V$ in a WM ROI (red circle) (D). A slight increase of the $C V$ in the segmented WM is visible at higher $V_{\text {ref }}$, possibly reflecting PVEs (E). The solid lines in the scatter plots represent the respective MPRAGE values before normalization.

\subsection{Implementation at Different Resolutions}

Figure 3 shows normalized MPRAGE images of the same subject for 0.7 and 0.9-mm isotropic resolution (without elliptical phase encoding). The histograms indicate that signal intensity in GM remains relatively unchanged while WM becomes brighter when the TF is reduced, thus increasing the WM-GM contrast. The reduction in contrast due to higher TF is in concordance with findings by Deichmann et al. [7] No increase in Gibbs ringing at the lower spatial resolution was observed. 


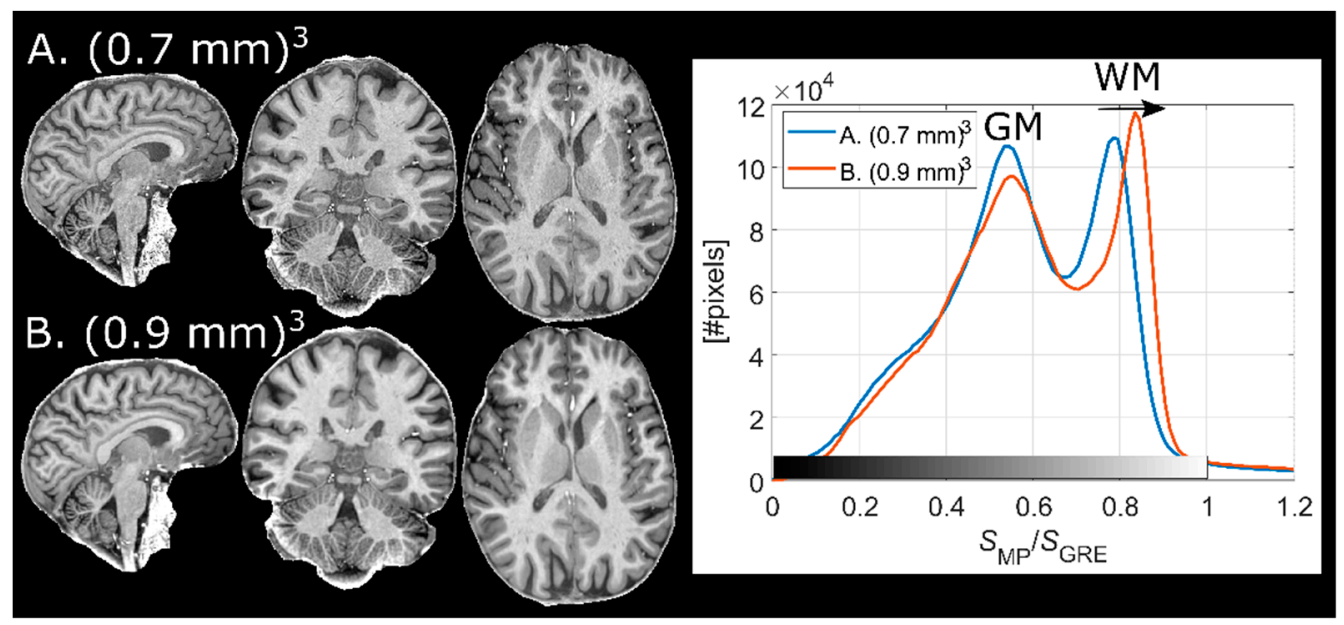

Figure 3. Normalized MPRAGE images acquired on the same subject with two protocols with different resolutions. (A) $(0.7 \mathrm{~mm})^{3}$, and (B) $(0.9 \mathrm{~mm})^{3}$. At $0.9 \mathrm{~mm}$ isotropic resolution, WM pixel values are increased (black arrow in histogram plot) relative $(0.7 \mathrm{~mm})^{3}$ while GM pixel values remain largely unaffected, increasing tissue contrast. This is an effect of the shortened readout train (lower TF).

\subsection{Intra-Subject Variability}

The MPRAGE volumes, before and after normalization, are shown in Figure 4. The normalized volumes show a noticeably increased spatial homogeneity. Hyperintense pixel values in the temporal lobes and cerebellum of the normalized volumes are indicative of a failed inversion with the adiabatic pulse [24]. These bright pixel intensities were exacerbated in session \#4, possibly due to a transmitter adjustment failure. Before normalization, the average CVs were $20 \pm 7.8 \%$ in WM, $32 \pm 12 \%$ in GM and $46 \pm 19 \%$ in CSF (Figure 5). The corresponding values after normalization were $7.9 \pm 3.3 \%$ in WM, $15 \pm 7.0 \%$ in GM and $33 \pm 16 \%$ in CSF. Exclusion of session \#4 yielded an average $C V$ of $16 \pm 8.1 / 27 \pm 14 / 39 \pm 24 \%$ in WM/GM/CSF before normalization and $7.2 \pm 2.5 / 11 \pm 6.8 / 22 \pm 14 \%$ after. The results of the ROI-based analysis can be seen in Table 2.

\subsection{Inter-Subject Variability}

The $C V$ was substantially reduced after normalization also in the inter-subject comparison (Figure 5). This improved reproducibility was most evident in the WM and basal ganglia since the diffeomorphic registration made a pixelwise comparison difficult in the cortex. Before normalization, the average $C V$ s in MNI space were $13 \pm 7.8 / 22 \pm 9.5 / 45 \pm 20 \%$ in $\mathrm{WM} / \mathrm{GM} / \mathrm{CSF}$, respectively. The corresponding values after normalization were $7.6 \pm 7.6 / 18 \pm 10 / 43 \pm 24 \%$. Based on individual segmentations, the $C V$ s were $6.2 / 6.5 / 9.0 \%$ before normalization and 2.0/4.3/6.4\% after in WM/GM/CSF. Results of the ROI-based analysis can be seen in Table 2 .

Table 2. ROI analysis of the $C V$ maps in Figure 5.

\begin{tabular}{ccccc}
\hline ROI & $\begin{array}{c}\text { Intra-Subject } \\
\text { before }\end{array}$ & $\begin{array}{c}\text { Intra-Subject } \\
\text { after } \\
\text { Normalization }\end{array}$ & $\begin{array}{c}\text { Inter-Subject } \\
\text { before }\end{array}$ & $\begin{array}{c}\text { Inter-Subject } \\
\text { after }\end{array}$ \\
\hline Frontal WM & $39 \pm 3.1 \%$ & $7.6 \pm 2.2 \%$ & $9.2 \pm 1.4 \%$ & $3.7 \pm 1.1 \%$ \\
Caudate head & $26 \pm 2.9 \%$ & $13 \pm 3.5 \%$ & $6.8 \pm 0.90 \%$ & $3.7 \pm 1.2 \%$ \\
Cerebellum & $21 \pm 3.6 \%$ & $8.7 \pm 2.9 \%$ & $22 \pm 1.4 \%$ & $4.8 \pm 0.91 \%$ \\
Temporal lobe & $34 \pm 4.3 \%$ & $9.8 \pm 2.3 \%$ & $16 \pm 1.8 \%$ & $6.1 \pm 1.3 \%$ \\
\hline
\end{tabular}




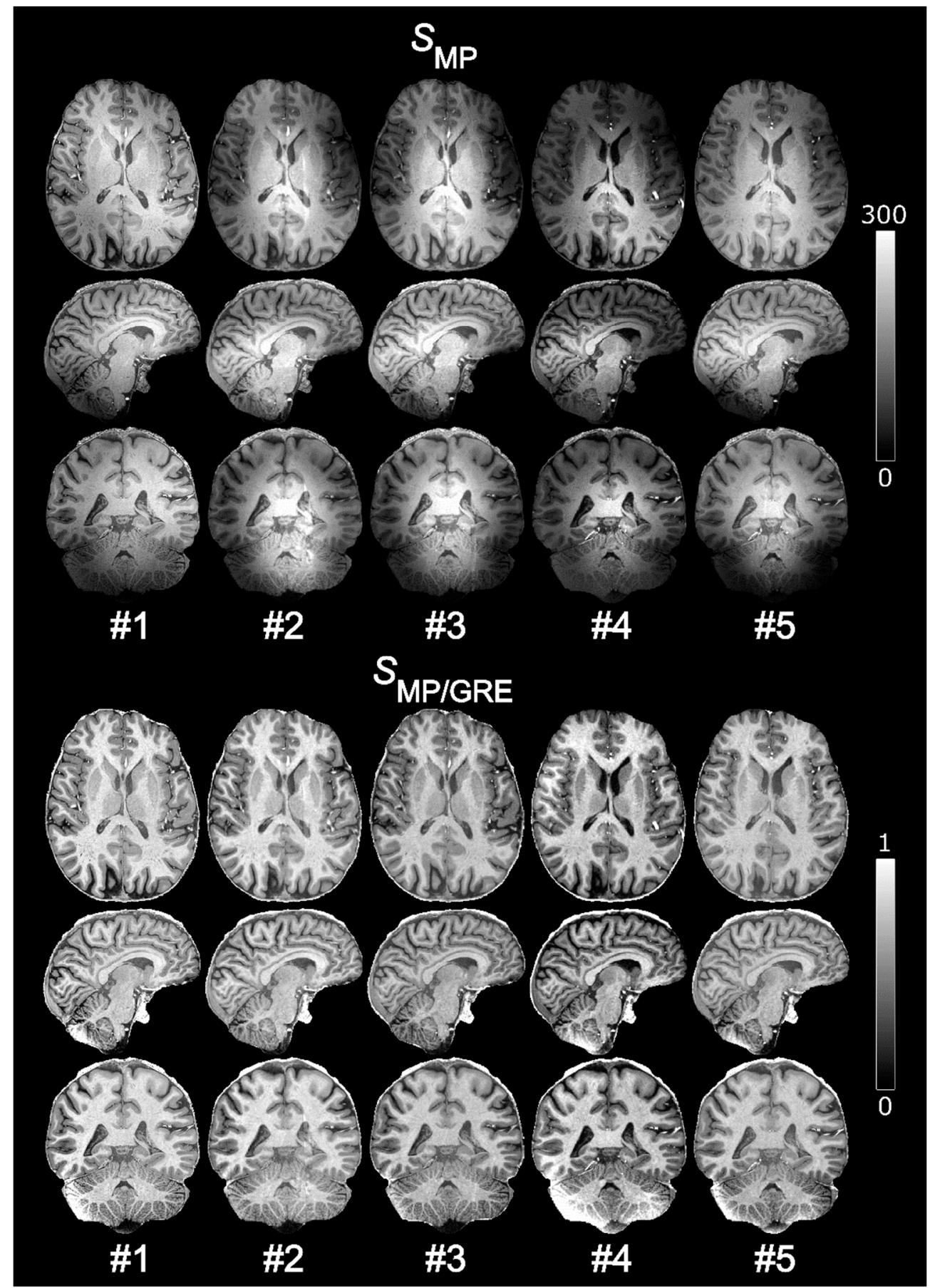

Figure 4. MPRAGE volumes before $\left(S_{\mathrm{MP}}\right)$ and after $\left(S_{\mathrm{MP} / \mathrm{GRE}}\right)$ normalization, acquired on a subject scanned at five separate sessions (day 1, 161, 170, 189, and 253). Influence from the bias field is noticeably reduced after normalization. Hyperintense pixels indicate a failed adiabatic inversion. At the 4th scanning session, there was possibly a transmitter adjustment failure, resulting in generally lower $B_{1}{ }^{+}$and thus a generally darker $S_{\mathrm{MP}}$ image as well as a larger area of very bright pixels in the cerebellumum of $S_{\mathrm{MP} / \mathrm{GRE}}$. 


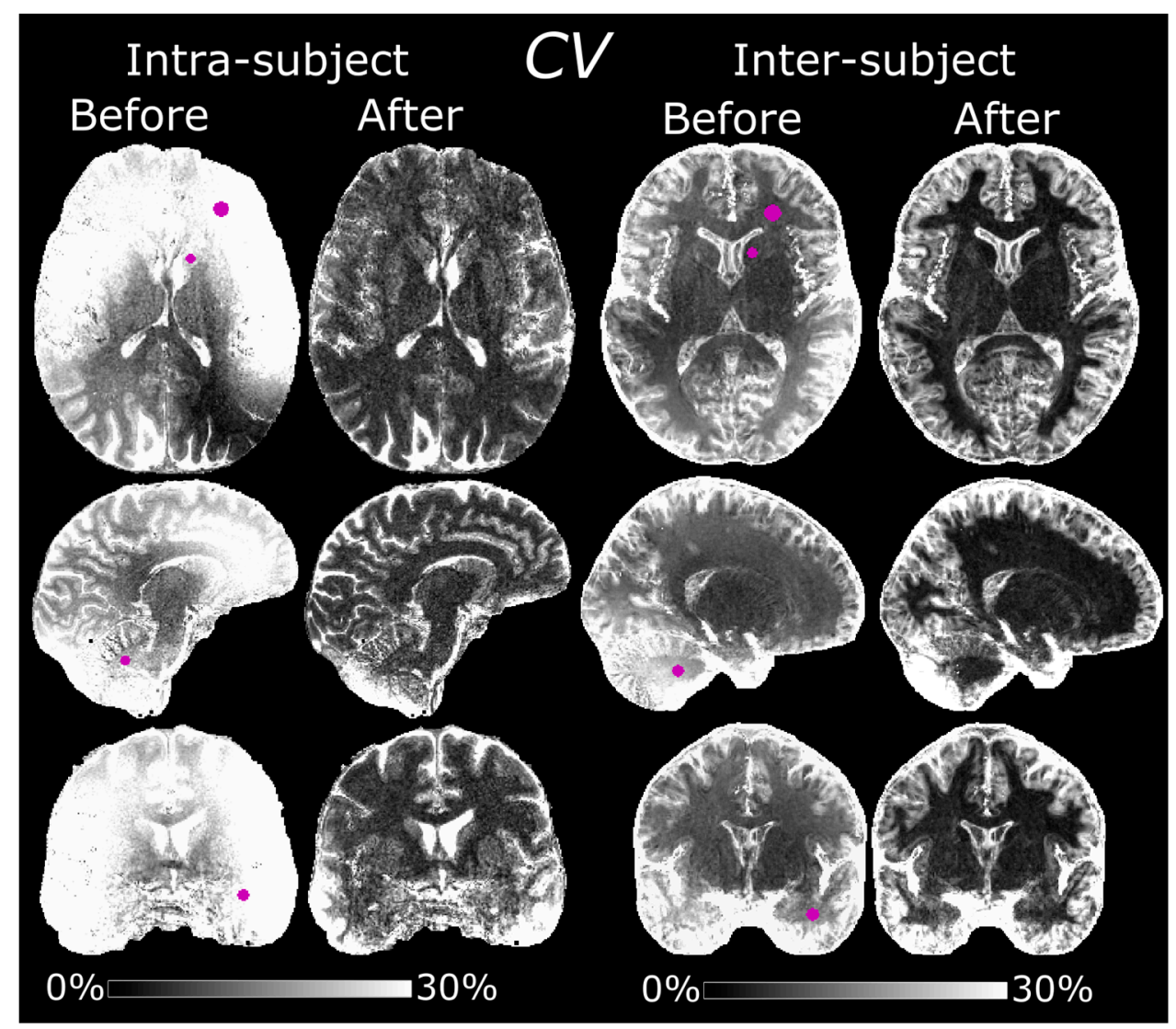

Figure 5. Maps of the $C V$ before and after normalization, showing the intra- and inter-subject variability. The intra-subject $C V$ maps were calculated from data acquired at five scanning sessions under an eight-month period. The inter-subject $C V$ maps, in MNI space, were calculated from data acquired from 10 separate subjects. Reproducibility was improved after normalization in both cases. The ROIs corresponding to the data in Table 2 are denoted in purple in the "Before" maps.

\subsection{Comparison to MP2RAGE}

The MP2RAGE image showed a higher WM-GM contrast than the normalized MPRAGE obtained using the $(0.7 \mathrm{~mm})^{3}$ protocol suggested here (Figure 6). The difference in pixel intensity between the WM/GM histogram modes was $0.785-0.545=0.24$ for the normalized MPRAGE compared to $0.275-(-0.155)=0.43$ for the MP2RAGE. The underlying reason is the larger dynamic range of $M_{z}$ in MP2RAGE. The interleaved acquisition and complex image combination of the MP2RAGE approach can utilize this as information of the polarity of $M_{z}$ is retained. The interleaved acquisition of the reference GRE, however, requires a longer $T_{\text {cycle, }}$ which was $5.0 \mathrm{~s}$ for MP2RAGE compared to $3.5 \mathrm{~s}$ in the sequential protocol. This in turn entails a longer $T_{\text {acq, }}$, which in this case was 8:20 min compared to $5: 59+1: 10=7: 09 \mathrm{~min}$. The acquisition time of the sequential protocol could have been reduced further to 4:35 +0:54 =5:29 $\mathrm{min}$ if an elliptical k-space encoding (as was used for the MP2RAGE sequence) had been employed. 


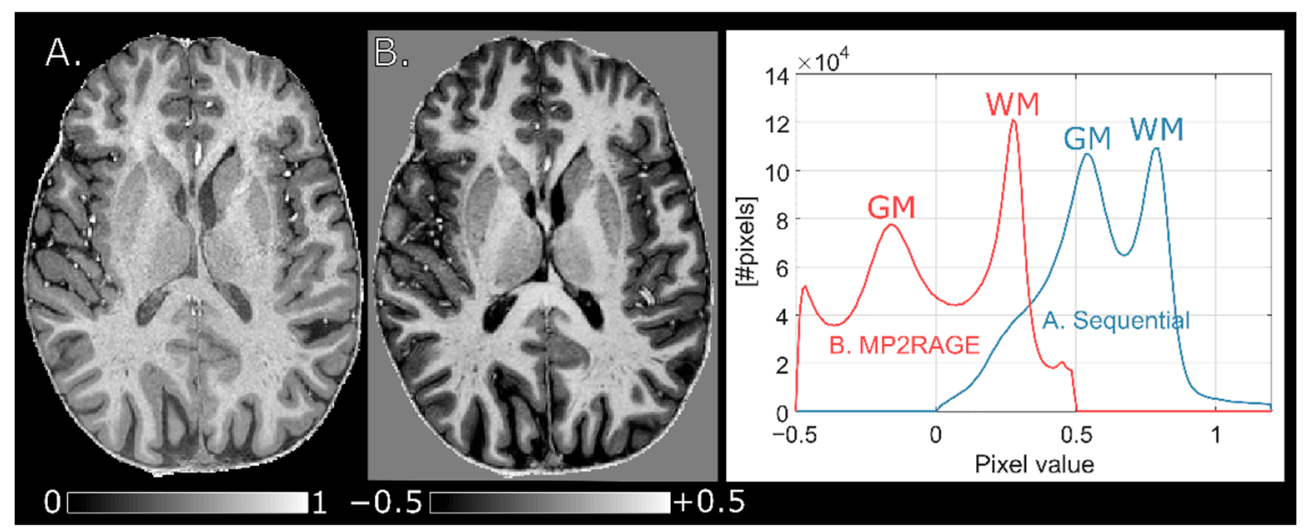

Figure 6. A normalized MPRAGE image (A) compared to an MP2RAGE image (B) with comparative whole-brain histograms. The MP2RAGE utilizes a larger dynamic range through the interleaved acquisition and complex combination and thus has a higher WM-GM contrast.

\section{7. $T_{1}$ Calculation}

The evolution of $\widetilde{M}_{z, M P}$ over $T_{\text {cycle }}$ for TF $=288$ is shown in panel A of Figure 7. The LUT signals were derived from this evolution for different $T_{1} \mathrm{~s}$ and $f_{\mathrm{T}}=1$ (panel B). The resulting $T_{1}$ as a function of $S_{\text {MP2RAGE }}$ at different $f_{\mathrm{T}}$ values is also shown (panel C). For these sequence parameters, the $B_{1}{ }^{+}$bias was most pronounced at long $T_{1}$ (i.e., for CSF). Around approximately $1000 \mathrm{~ms}$, however, $T_{1}$ was no longer uniquely defined for a given $S_{\text {MP2RAGE }}$, which thus set the lower limit of measurable $T_{1}$. This lower limit varied, based on $f_{\mathrm{T}}$ as $1122 / 1156 / 1181 / 1169 / 1097 / 966 / 810 \mathrm{~ms}$ for $f_{\mathrm{T}}=0.4 / 0.6 / 0.8 / 1.0 / 1.2 / 1.4 / 1.6$, respectively. At $f_{\mathrm{T}}=0.4$, there was also an upper limit at $3330 \mathrm{~ms}$. Figure 8 shows a map of $T_{1}$ derived from this LUT before (panel A) and after (panel B) $B_{1}{ }^{+}$-correction. Estimated tissue $T_{1}$ was only moderately affected by the $B_{1}{ }^{+}$-correction although an elevation of $T_{1}$ in the thalamus $\left(f_{\mathrm{T}} \approx 1.3\right)$ after correction can be discerned. The most notable difference was seen in the CSF, which was adjusted toward higher values in high $B_{1}{ }^{+}$areas and toward lower values in low $B_{1}{ }^{+}$areas. Especially after correction, the distribution of $T_{1}$ seemed to be very homogenous within different tissues, without any obvious $B_{1}{ }^{+}$bias.

\subsection{Example Results Using the Finalized Protocol}

Figure 9 shows images from a subject using the finalized protocol $\left(0.8^{3} \mathrm{~mm}^{3}\right.$, without elliptical phase encoding). After normalization, effects from the intensity field bias were considerably reduced. For instance, the diagonal $B_{1}{ }^{+}$pattern in the axial plane was removed as well as the right-left asymmetry in the coronal plane. The improved homogeneity can also be seen in the whole-brain histogram where WM and GM form distinct modes after normalization. Issues of extremely low $B_{1}{ }^{+}\left(f_{\mathrm{T}} \sim 0.3\right)$ in the right part of the cerebellum, where the local flip angle is too low to fulfil the adiabatic condition for inversion, can, however, not be resolved by normalization (red arrow). 
A

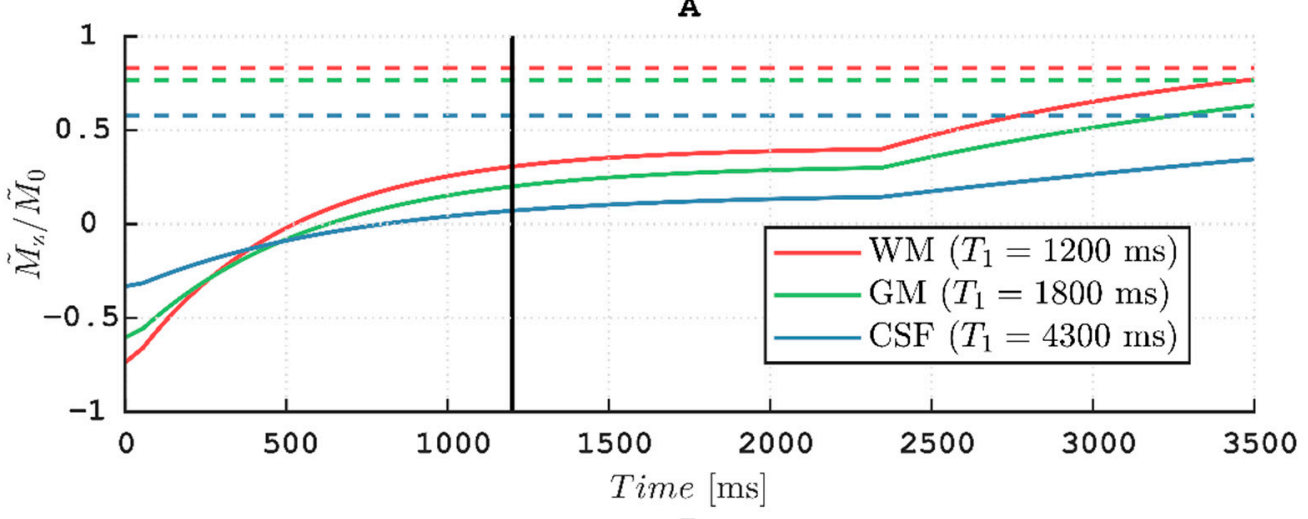

B
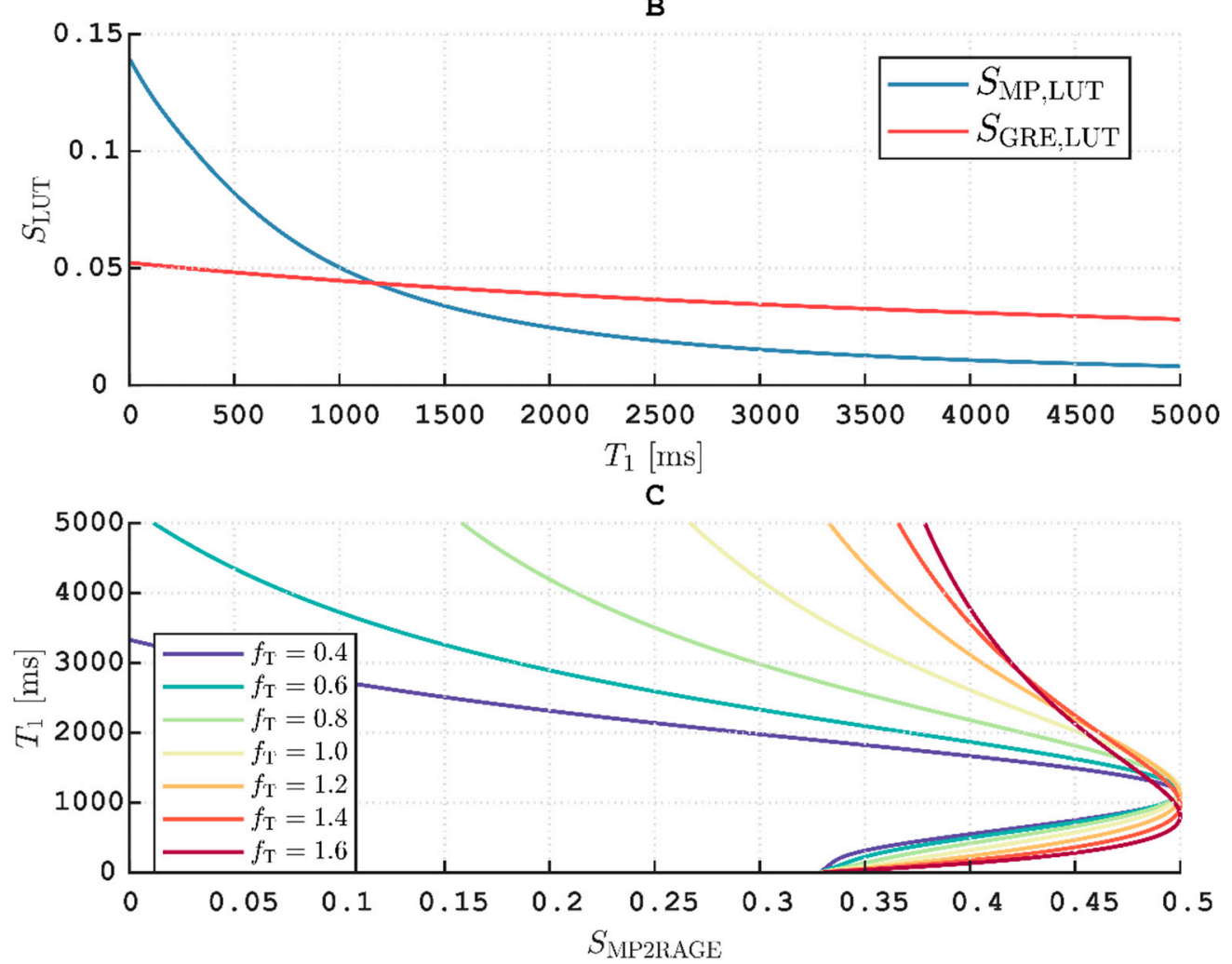

Figure 7. (A) Solid coloured lines show the evolution of $M_{Z}$ during an MPRAGE acquisition across $T_{\text {cycle }}=3500 \mathrm{~ms}$ and $f_{\mathrm{T}}=1$ for three values of $T_{1}$ exemplifying WM, GM, and CSF. The dashed lines show the corresponding steady-state of the reference GRE. Vertical black line denotes the center of k-space at TI $=1200 \mathrm{~ms}$. (B) The two LUT signals as a function of $T_{1}$ for $f_{\mathrm{T}}=1$. (C) Estimated $T_{1}$ as a function of $S_{M P 2 R A G E}$ for different $f_{\mathrm{T}}$. Areas with longer $T_{1}$ were disproportionately biased by deviations in $f_{\mathrm{T}}$. Depending on $f_{T}$, the minimum $T_{1}$ that could be uniquely defined ranged from $1181 \mathrm{~ms}\left(f_{\mathrm{T}}=0.8\right)$ and $810 \mathrm{~ms}\left(f_{\mathrm{T}}=1.6\right)$. At $f_{\mathrm{T}}=0.4$, there was also an upper limit at $3330 \mathrm{~ms}$. 


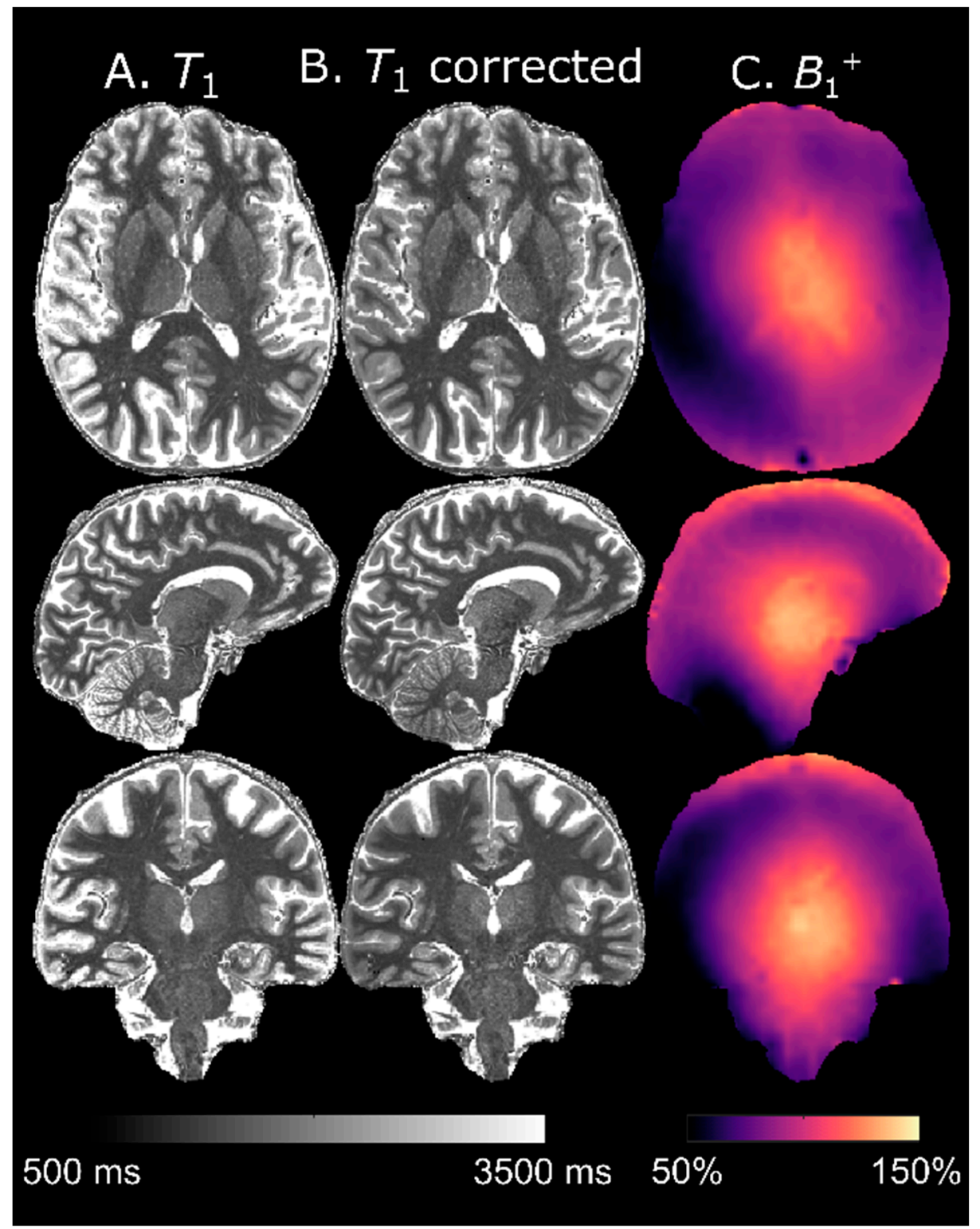

Figure 8. LUT-derived $T_{1}$ maps, uncorrected (A) and corrected (B) with a separately acquired $B_{1}{ }^{+}$ map (C). The $T_{1}$ estimation in tissue was moderately robust against $B_{1}{ }^{+}$influence and mostly CSF was affected. 


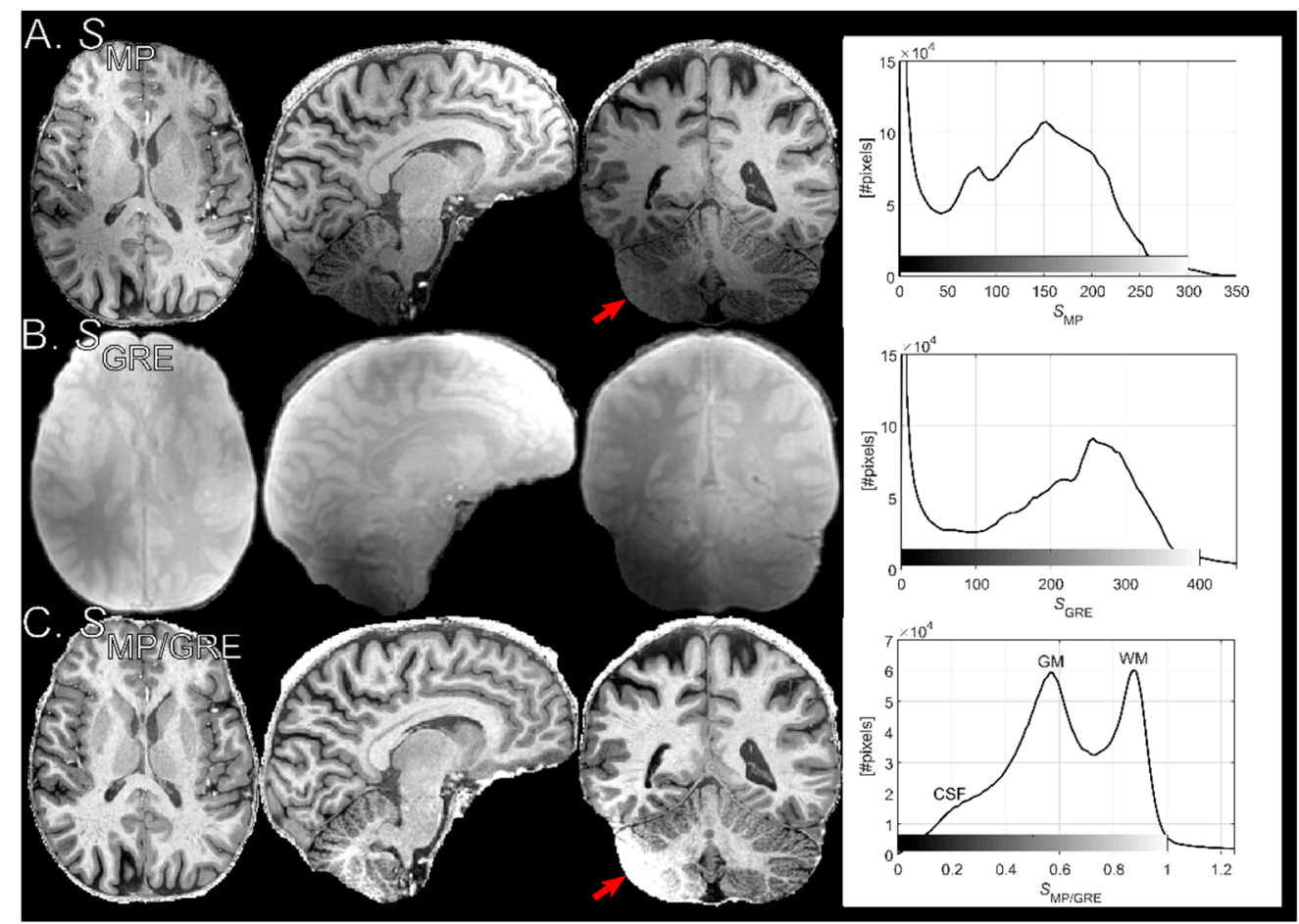

Figure 9. Example images acquired with the MPRAGE protocol before normalization (A), the reference GRE used for normalization (B), and the MPRAGE image after normalization (C). The righthand panel shows whole-brain histograms of the respective images. Normalization clearly reduced spatial heterogeneity from $B_{1}$. The improved homogeneity is also illustrated by the histograms, where GM and WM (and to a lesser extent CSF) modes are visible after normalization. In the right part of the cerebellum (red arrow, coronal plane), $B_{1}{ }^{+}$is very weak, leading to failed adiabatic inversion.

\section{Discussion}

In this study, we describe the process of implementing a sequential protocol for bias field correction of MPRAGE images at $7 \mathrm{~T}$. Effects of varying the flip angle, different spatial resolutions and acquisition voxel size of the reference GRE were studied, mainly to improve WM-GM contrast and to minimize scan time without introducing biases. The main purpose of the protocols was to obtain semi-quantitative images with "pure" $T_{1}$ contrast and improved reproducibility. Improved intra-subject reproducibility was demonstrated by a decreased $C V$ of $7.9 \pm 3.3 \%$ in segmented WM after normalization compared to $20 \pm 7.8 \%$ before. Likewise, an improved inter-subject reproducibility was demonstrated by the $C V$ in segmented WM of 10 subjects which decreased from $13 \pm 7.8 \%$ to $7.6 \pm 7.6 \%$. Local improvement could be higher, for instance $39 \pm 3.1 \%$ to $7.6 \pm 2.2 \%$ in frontal WM in the intra-subject experiment and $22 \pm 1.4 \%$ to $4.4 \pm 0.91 \%$ in the cerebellum in the inter-subject experiment.

The multiplicative spatial intensity bias imposed by the combination of receive coil signals was removed by normalization. Due to a smaller readout flip angle in the reference GRE compared to the MPRAGE, and to the rather small flip angles overall, the effect of the inhomogeneous transmit field $\left(B_{1}{ }^{+}\right)$was also mostly removed. Since the analytical description of the MPRAGE signal is too complicated to evaluate [25], we estimated $T_{1}$ maps through a LUT-based approach analogous to MP2RAGE [5]. As expected, accuracy was improved by using a separate $B_{1}{ }^{+}$map.

The protocol was not designed with $T_{1}$ calculation, as featured by MP2RAGE, in mind. Thus, at the rather long TI $=1200 \mathrm{~ms}$ the protocol fails to effectively exploit the dynamic range obtained when inverting fully relaxed longitudinal magnetization (cf. Figure 7, panel A). This results in quite "saturated" images when normalizing using Equation (8) (pixels close to 0.5 ), leading to a loss of precision in the $T_{1}$ calculation. Further, although whole-brain histograms of $T_{1}$ were very similar when using different $V_{\text {ref, }}$, it is most likely 
prudent to use identical voxel sizes of MPRAGE and the reference GRE if accurate $T_{1}$ estimation is of interest, especially at the cortical boundaries that are most susceptible to PVEs. It is important to note that the LUT-based approach assumes that all differences in pixel values are solely caused by variations in $T_{1}$ (i.e., that there is "pure" $T_{1}$ contrast). By design, the influence of $B_{1}{ }^{+}$inhomogeneities on the $T_{1}$ calculation is decreased by the normalization of signals. The choice of $\alpha_{\mathrm{MP}}=2.7 \alpha_{\mathrm{GRE}}\left(8^{\circ} \mathrm{vs} .3^{\circ}\right)$ appeared to minimize residual effects of $B_{1}{ }^{+}$inhomogeneity on the normalized MPRAGE (cf. Figure 1) and thus also on the $T_{1}$ calculation. The largest residual effect of $B_{1}{ }^{+}$inhomogeneity was found at $\alpha_{\mathrm{MP}}=1.3 \alpha_{\mathrm{GRE}}\left(8^{\circ} \mathrm{vs} .6^{\circ}\right)$. This is in concordance with the work of Van de Moortele et al. in which a choice of $\alpha_{\mathrm{MP}}=\alpha_{\mathrm{GRE}}$ resulted in a much stronger residual $B_{1}{ }^{+}$dependence than $\alpha_{\mathrm{MP}}=2 \alpha_{\mathrm{GRE}}$ [4]. To increase accuracy, a separately acquired flip angle map is still recommended if $T_{1}$-mapping is of interest [26]. This is particularly important for longer $T_{1}$ values where the $B_{1}{ }^{+}$influence is stronger (cf. panel C, Figure 7). The loss of contrast observed in the cerebellum (cf. panel C, Figure 9) occurs when $B_{1}{ }^{+}$decreases below the threshold required for the inversion pulse to fulfil the adiabatic condition which cannot be resolved by flip angle mapping [24].

The obvious benefit of an interleaved acquisition is that identical scanning conditions, such as RF power calibration, is guaranteed, as well as increased robustness against interscan movement. Inter-scan motion can be corrected by offline rigid coregistration, however. Further, the reduced duration of any one acquisition will reduce the total risk of intra-scan subject movement. The risk of introducing $T_{1}$ contrast in the GRE reference due to poor timings (mainly TI and/or TF $\times$ TR being too short) is also removed [4]. Importantly, the option to increase $V_{\text {ref }}$ facilitates the possibility to have a shorter total scan time than needed for the interleaved MP2RAGE.

A protocol with an MPRAGE acquisition voxel size of $(0.6 \mathrm{~mm})^{3}$ was also explored (data not shown). However, the SNR in the MPRAGE was deemed unacceptably low. Hence, the $(0.7 \mathrm{~mm})^{3}$ protocol here represents the upper limit of the spatial resolution imposed by SNR. It should be noted that noise propagation will moderately decrease the SNR in the normalized MPRAGE, $S_{\mathrm{MP} / \mathrm{GRE}}$, relative to the unnormalized, $S_{\mathrm{MP}}$, by $\frac{S_{\mathrm{MP}}}{\sqrt{1+S_{\mathrm{MP} / \mathrm{GRE}}^{2}}}$, thereby somewhat adversely affecting obtainable spatial resolution [4]. Decreasing the acquisition voxel size also entails increasing TF and thus the duration of the readout train and eventually of TI. This limitation can be circumvented by introducing a SENSE factor in the inner loop, as shown here for $(0.7 \mathrm{~mm})^{3}$. The employed zigzag k-space trajectory (see the view-ordering schemes presented for turbo spin echoes in ref. [27]) allows the use of a TF larger than the number of inner loop k-space lines $\left(N_{y}\right)$, which is an effective way to decrease scan time. It further allows the enabling of an elliptical k-space phase encoding which decreases the acquisition time by a factor of approximately $r^{2} /\left(\pi(r / 2)^{2}\right) \approx 1.3$ (cf. Table 1). To our knowledge, this kind of k-space trajectory is only readily available on Philips's systems. Hence, the total scan time of the protocols are supplied with/without elliptical phase encoding.

Correction of RF field-induced bias is recommended at field strengths of $7 \mathrm{~T}$ and above. However, normalization may also be necessary to correct for intensity field bias at $3 \mathrm{~T}$, as the wavelength of the $B_{1}$ field is approximately $25 \mathrm{~cm}$ in brain tissue and thus of the same order of magnitude as the imaged object [28].

\section{Limitations}

We did not attempt to optimize the point-spread function (PSF) of the MPRAGE sequence, although this will be broadened when signal is acquired during a transient state [8]. At increasing resolution, readouts are appended at the beginning and end of the RAGE trains (cf. Figure 7, panel A). These will compromise the PSF, so the effective spatial resolution will not increase as much as the nominal spatial resolution. The increased change in $M_{z}$ during acquisition may also influence the WM-GM contrast (cf. Figure 3). In this work, we focused on the reference GRE for normalization, which is acquired entirely in 
a steady-state and thus does not suffer from such PSF distortions. Further, the PSF cannot be modelled for an elliptical k-space phase encoding, since the trajectory is proprietary.

Like in MP2RAGE, the loss of $T_{1}$ contrast at very low $B_{1}{ }^{+}$(below the threshold for adiabatic inversion) cannot be mended by normalization [22].

Although subdural ringing artifacts did not noticeably increase when using a protocol with lower spatial resolution (cf. Figure 3), curved ringing artifacts were occasionally observed. These were more evident in the reference GRE but could also be seen in MPRAGE. The artifacts appeared to be correlated to subject movement, but this was not confirmed. These artifacts are believed to be related to interpolation of the low spatial resolution $\left(5.5 \times 7.4 \times 4.0 \mathrm{~mm}^{3}\right)$ SENSE reference scan, acquired prior to the MPRAGE and reference GRE. The artifact was very similar to the "streaky-linear" artifact "type A" described by Sartoretti et al. and showcased in Figure 3, panels (g), (h), and (i) [29].

\section{Conclusions}

We describe a sequential protocol for correction of RF-induced bias in MPRAGE images by normalization with a reference GRE. Spatial homogeneity and WM-GM contrast were improved after normalization as well as intra- and inter-subject reproducibility. Scan time could be reduced by increasing the voxel size of the reference GRE without appreciably affecting image quality.

Author Contributions: Conceptualization, G.H.; methodology, G.H. and H.O.; software, H.O. and J.L.; validation, H.O.; formal analysis, H.O.; investigation, G.H. and H.O.; data curation, G.H., H.O., and M.N.; writing—original draft preparation, H.O.; writing—review and editing, H.O., G.H., R.W., J.L., and M.N.; visualization, H.O.; supervision, G.H. and R.W.; funding acquisition, G.H. and R.W. All authors have read and agreed to the published version of the manuscript.

Funding: This research was funded by the Swedish Research Council, grant numbers NT-2014-6193 and M-2017-00995. The APC was funded in part by Lund University.

Institutional Review Board Statement: The study was conducted according to the guidelines of the Declaration of Helsinki, and approved by the Regionala Etikprövningsnämden Lund (9 August 2012, protocol code 2012/428).

Informed Consent Statement: Written informed consent was obtained from all subjects prior to each examination.

Data availability statement: Data is openly available at https:/ / openneuro.org/datasets/ds003769 (accessed on 10 September 2021). The inter-subject variability data is available as sub-001 to sub-010 at https:/ / openneuro.org/datasets / ds003508 (accessed on 10 September 2021). Descriptive pulse sequence text files as well as a MATLAB scripts to perform the $T_{1}$ calculation is available at https: //github.com/OlssonHampus/RF_bias_correction_MPRAGE (accessed on 10 September 2021).

Acknowledgments: The authors are indebted to Fredy Visser, Philips Healthcare, for providing the initial MPRAGE protocol. The Lund University Bioimaging Center (LBIC) is acknowledged for experimental resources. The publication was approved in its submitted form by Philips Medical Systems Nederland B.V. in accordance with the research agreement with LBIC.

Conflicts of Interest: The authors declare no conflict of interest. The funders had no role in the design of the study; in the collection, analyses, or interpretation of data; in the writing of the manuscript, or in the decision to publish the results.

\section{References}

1. Mugler, J.P., 3rd; Brookeman, J.R. Three-dimensional magnetization-prepared rapid gradient-echo imaging (3D MP RAGE). Magn. Reson. Med. 1990, 15, 152-157. [CrossRef]

2. Balchandani, P.; Naidich, T.P. Ultra-High-Field MR Neuroimaging. AJNR Am. J. Neuroradiol. 2015, 36, 1204-1215. [CrossRef] [PubMed]

3. Focke, N.K.; Helms, G.; Kaspar, S.; Diederich, C.; Toth, V.; Dechent, P.; Mohr, A.; Paulus, W. Multi-site voxel-based morphometryNot quite there yet. Neuroimage 2011, 56, 1164-1170. [CrossRef] 
4. Van de Moortele, P.F.; Auerbach, E.J.; Olman, C.; Yacoub, E.; Ugurbil, K.; Moeller, S. T1 weighted brain images at 7 Tesla unbiased for Proton Density, T2* contrast and RF coil receive B1 sensitivity with simultaneous vessel visualization. Neuroimage 2009, 46, 432-446. [CrossRef] [PubMed]

5. Marques, J.P.; Kober, T.; Krueger, G.; van der Zwaag, W.; Van de Moortele, P.F.; Gruetter, R. MP2RAGE, a self bias-field corrected sequence for improved segmentation and T1-mapping at high field. Neuroimage 2010, 49, 1271-1281. [CrossRef] [PubMed]

6. Trampel, R.; Bazin, P.L.; Pine, K.; Weiskopf, N. In-vivo magnetic resonance imaging (MRI) of laminae in the human cortex. Neuroimage 2019, 197, 707-715. [CrossRef]

7. Deichmann, R.; Good, C.D.; Josephs, O.; Ashburner, J.; Turner, R. Optimization of 3-D MP-RAGE sequences for structural brain imaging. Neuroimage 2000, 12, 112-127. [CrossRef]

8. Mugler, J.P., 3rd; Brookeman, J.R. Rapid three-dimensional T1-weighted MR imaging with the MP-RAGE sequence. J. Magn. Reson. Imaging 1991, 1, 561-567. [CrossRef]

9. Teeuwisse, W.M.; Brink, W.M.; Webb, A.G. Quantitative assessment of the effects of high-permittivity pads in 7 Tesla MRI of the brain. Magn. Reson. Med. 2012, 67, 1285-1293. [CrossRef]

10. Pruessmann, K.P.; Weiger, M.; Scheidegger, M.B.; Boesiger, P. SENSE: Sensitivity encoding for fast MRI. Magn. Reson. Med. 1999, 42, 952-962. [CrossRef]

11. Li, X.; Morgan, P.S.; Ashburner, J.; Smith, J.; Rorden, C. The first step for neuroimaging data analysis: DICOM to NIfTI conversion. J. Neurosci. Methods 2016, 264, 47-56. [CrossRef]

12. Chenevert, T.L.; Malyarenko, D.I.; Newitt, D.; Li, X.; Jayatilake, M.; Tudorica, A.; Fedorov, A.; Kikinis, R.; Liu, T.T.; Muzi, M.; et al. Errors in Quantitative Image Analysis due to Platform-Dependent Image Scaling. Transl. Oncol. 2014, 7, 65-71. [CrossRef]

13. Jenkinson, M.; Bannister, P.; Brady, M.; Smith, S. Improved optimization for the robust and accurate linear registration and motion correction of brain images. Neuroimage 2002, 17, 825-841. [CrossRef]

14. Jenkinson, M.; Beckmann, C.F.; Behrens, T.E.; Woolrich, M.W.; Smith, S.M. Fsl. Neuroimage 2012, 62, 782-790. [CrossRef]

15. Smith, S.M. Fast robust automated brain extraction. Hum. Brain Mapp. 2002, 17, 143-155. [CrossRef]

16. Zhang, Y.; Brady, M.; Smith, S. Segmentation of brain MR images through a hidden Markov random field model and the expectation-maximization algorithm. IEEE Trans. Med. Imaging 2001, 20, 45-57. [CrossRef]

17. Rooney, W.D.; Johnson, G.; Li, X.; Cohen, E.R.; Kim, S.G.; Ugurbil, K.; Springer, C.S., Jr. Magnetic field and tissue dependencies of human brain longitudinal $1 \mathrm{H}_{2} \mathrm{O}$ relaxation in vivo. Magn. Reson. Med. 2007, 57, 308-318. [CrossRef] [PubMed]

18. Olsson, H.; Andersen, M.; Latt, J.; Wirestam, R.; Helms, G. Reducing bias in dual flip angle T1-mapping in human brain at 7T. Magn. Reson. Med. 2020, 84, 1347-1358. [CrossRef]

19. Noven, M.; Olsson, H.; Helms, G.; Horne, M.; Nilsson, M.; Roll, M. Cortical and white matter correlates of language-learning aptitudes. Hum. Brain Mapp. 2021. [CrossRef] [PubMed]

20. Ashburner, J. A fast diffeomorphic image registration algorithm. Neuroimage 2007, 38, 95-113. [CrossRef] [PubMed]

21. Tabelow, K.; Balteau, E.; Ashburner, J.; Callaghan, M.F.; Draganski, B.; Helms, G.; Kherif, F.; Leutritz, T.; Lutti, A.; Phillips, C.; et al. hMRI-A toolbox for quantitative MRI in neuroscience and clinical research. Neuroimage 2019. [CrossRef] [PubMed]

22. Helms, G.; Latt, J.; Olsson, H. Cross-vendor transfer and RF coil comparison of a high-resolution MP2RAGE protocol for brain imaging at 7T. Acta Sci. Lund. 2020, 2020,1-12.

23. Olsson, H.; Andersen, M.; Helms, G. Reducing bias in DREAM flip angle mapping in human brain at 7T by multiple preparation flip angles. Magn. Reson. Imaging 2020, 72, 71-77. [CrossRef]

24. Kadhim, M. Measuring T1 Using MP2RAGE in Human Brain at 7T-Effect of B1+ and Inversion Pulse Efficiency. Master's Thesis, Lund University, Lund, Sweden, 2021. Available online: https://lup.lub.lu.se/student-papers/search/publication/9043213 (accessed on 10 September 2021).

25. Wright, P.J.; Mougin, O.E.; Totman, J.J.; Peters, A.M.; Brookes, M.J.; Coxon, R.; Morris, P.E.; Clemence, M.; Francis, S.T.; Bowtell, R.W.; et al. Water proton T1 measurements in brain tissue at 7, 3, and $1.5 \mathrm{~T}$ using IR-EPI, IR-TSE, and MPRAGE: Results and optimization. Magn. Reson. Mater. Phys. Biol. Med. 2008, 21, 121-130. [CrossRef] [PubMed]

26. Haast, R.A.M.; Lau, J.C.; Ivanov, D.; Menon, R.S.; Uludag, K.; Khan, A.R. Effects of MP2RAGE B1(+) sensitivity on inter-site T1 reproducibility and hippocampal morphometry at 7T. Neuroimage 2021, 224, 117373. [CrossRef]

27. Busse, R.F.; Brau, A.C.; Vu, A.; Michelich, C.R.; Bayram, E.; Kijowski, R.; Reeder, S.B.; Rowley, H.A. Effects of refocusing flip angle modulation and view ordering in 3D fast spin echo. Magn. Reson. Med. 2008, 60, 640-649. [CrossRef] [PubMed]

28. Gabriel, C. Compilation of the Dielectric Properties of Body Tissues at RF and Microwave Frequencies; King's College: London, UK, 1996.

29. Sartoretti, T.; Reischauer, C.; Sartoretti, E.; Binkert, C.; Najafi, A.; Sartoretti-Schefer, S. Common artefacts encountered on images acquired with combined compressed sensing and SENSE. Insights Imaging 2018, 9, 1107-1115. [CrossRef] 